\title{
No Experimental Evidence of Co-Feeding Transmission of African Swine Fever Virus between Ornithodoros Soft Ticks
}

\author{
Rémi Pereira De Oliveira ${ }^{1,2,3}$ (), Evelyne Hutet ${ }^{3}$, Maxime Duhayon ${ }^{1,2}$, Frédéric Paboeuf ${ }^{3}$, \\ Marie-Frédérique Le Potier ${ }^{3} \mathbb{D}$ and Laurence Vial ${ }^{1,2, * \mathbb{D}}$ \\ 1 UMR ASTRE, CIRAD, Campus International de Baillarguet, F-34398 Montpellier, France; \\ remi.pereira_de_oliveira@cirad.fr (R.P.D.O.); maxime.duhayon@cirad.fr (M.D.) \\ 2 UMR ASTRE, Univ Montpellier, CIRAD, INRAE, Campus International de Baillarguet, \\ F-34398 Montpellier, France \\ 3 Swine Virology and Immunology Unit, Ploufragan-Plouzané-Niort Laboratory, ANSES, 35 rue de \\ Beaucemaine, 22440 Ploufragan, France; Evelyne.HUTET@anses.fr (E.H.); Frederic.PABOEUF@anses.fr (F.P.); \\ marie-frederique.lepotier@anses.fr (M.-F.L.P.) \\ * Correspondence: laurence.vial@cirad.fr
}

Received: 5 February 2020; Accepted: 27 February 2020; Published: 28 February 2020

\begin{abstract}
Ornithodoros soft ticks are the only known vector and reservoir of the African swine fever virus, a major lethal infectious disease of Suidae. The co-feeding event for virus transmission and maintenance among soft tick populations has been poorly documented. We infected Ornithodoros moubata, a known tick vector in Africa, with an African swine fever virus strain originated in Africa, to test its ability to infect $O$. moubata through co-feeding on domestic pigs. In our experimental conditions, tick-to-tick virus transmission through co-feeding failed, although pigs became infected through the infectious tick bite.
\end{abstract}

Keywords: African swine fever; Argasid tick; co-feeding; Ornithodoros moubata

\section{Introduction}

African swine fever (ASF) is a lethal disease of Suidae caused by the African swine fever virus (ASFV). ASF is endemic in southern and eastern Africa, where ASFV circulates among different hosts, such as domestic pigs and warthogs, as well as soft tick vectors from the Ornithodoros moubata group [1-4]. Three main transmission cycles are commonly described in these endemic areas: (i) the sylvatic cycle, corresponding to ASFV transmission between warthogs and ticks, (ii) the domestic/tick cycle, corresponding to ASFV transmission between domestic pigs and ticks, and (iii) the domestic cycle, corresponding to ASFV circulation between domestic pigs only [5,6]. It is assumed that soft ticks play an important role in maintaining ASFV within the sylvatic cycle, as well as in transmitting ASFV between wild and domestic Suidae $[4,7,8]$.

To be infected, ticks need to engorge on viremic animals with a sufficient viral load [9]. As viremia in warthogs is typically low (or undetectable), Plowright (1977) assumed that viremia in warthogs could not be sufficient to infect soft ticks [10]. He concluded that warthogs may only act as carriers of infected soft ticks, thanks to their movements between wild and domestic areas [10]. Conversely, Thomson (1980) conducted experimental infections of young warthogs, that are most commonly reported to be infected in the wild, and also the only stage that remains in close contact with endophilous soft ticks in burrows [11]. Against all expectations, those warthogs developed high viremia, at least for two weeks, which was considered sufficient to infect soft ticks. The use of new-born warthogs might explain the higher virus titers observed because of the immunological immaturity of the animals [11]. To date, 
the role of the sylvatic cycle between warthogs and soft ticks in maintaining ASFV in African wild areas has remained unclear. At the same time, other studies have demonstrated that soft ticks can also become infected without vertebrate hosts through ASFV vertical transmission to descendants $[9,12]$ and ASFV sexual transmission between males and females during copulation [13], at least with some tick species and viral strains.

Apart from such tick-to-tick transmission modes, co-feeding might also be assumed to be an alternative or complementary route of ASFV transmission to sexual and vertical transmission in this specific context. Indeed, co-feeding is described as an efficient non-systematic transmission pathway allowing tick infection through biting on hosts with low or no viremia. It occurs when infected and uninfected ticks take their blood meal in spatio-temporal proximity on the same non-viremic $[14,15]$ or immunized vertebrate host [16]. This mode of transmission has been demonstrated in hard ticks for certain viruses, such as the Thogoto virus [17], the Tick-borne encephalitis virus [18], the Crimean-Congo hemorrhagic fever virus [19], and for bacteria such as Borrelia afzelli [20]. To our knowledge, only one study conducted by Rennie (1998) has evaluated co-feeding as another pathway for ASFV dissemination among soft tick populations. However, the results were not conclusive [21].

In our study, we experimentally assess the ASFV transmission through co-feeding, using infected and uninfected O. moubata ticks and an ASFV strain originally isolated from the same tick species in the wild [22]. This study is part of investigations testing different pathways of ASFV transmission by soft ticks.

\section{Materials and Methods}

The animal experiments were authorized by the French Ministry for Research (project No. 2017062615498464) and approved by the national ethics committee (authorization No. 11/07/17-3).

The Liv13/33, a hemadsorbing ASFV strain (genotype I), used for tick infections was isolated from O. moubata in Livingstone park, Zambia in 1983 [22] and was kindly provided by Dr. Linda Dixon (OIE reference laboratory, Pirbright Institute, UK).

The O. moubata ticks ("Neuchâtel strain") used in the experiment were O. moubata sensu stricto, as described by Bakkes et al. (2018) [1]. They originated from Southern Africa and were maintained in the Neuchâtel University insectary for at least twenty years, and then reared at CIRAD since 2008.

Males and females of $O$. moubata were infected with the Liv13/33 strain by blood feeding on viremic pigs with a viremia ranging from $10^{7,8}$ hemadsorbing dose $50 \% / \mathrm{mL}\left(\mathrm{HAD}_{50} / \mathrm{mL}\right)$ to $10^{8,1} \mathrm{HAD}_{50} / \mathrm{mL}$, as previously described [23]. The resulting O. moubata- Liv13/33 tick-virus pair (OmL) was used as the "source" for the co-feeding transmission trial 8 months post infection. OmL was previously confirmed to be competent to transmit ASFV to pigs through biting, 2 and 8 months post infection [24]. Furthermore, all OmL tested by real-time PCR were highly positive for ASFV [24].

Uninfected O. moubata ticks (OmCF), at the first nymphal development stage (nymphs-1), were used as "recipient" for the co-feeding transmission trial. Nymphs-1 were preferred, so as to be easily distinguished after engorgement from the infected "source" adult ticks.

The co-feeding transmission trial was conducted using two replicates: 1) eight infected females and seven infected males mixed with 30 uninfected nymphs-1 (group 1), and 2) nine infected females and six infected males mixed with 30 uninfected nymphs-1 (group 2). Each of the two groups were fed at the same time on the same specific pathogen-free (SPF) pig (7 weeks-old Large White female) in the same Petri dish ( $6 \mathrm{~cm}$ diameter) for 3 hours. Petri dishes were fixed to the pig's abdomen with a bandage. The abdomen zone was chosen because the skin is thin, which eases the engorgement of ticks.

After engorgement, the ticks were washed to prevent external contamination and then sorted into "engorged" versus "non-engorged" ticks, distinguishing between adults and nymphs-1 (Table 1). Ticks' feces and coxal fluid, excreted and secreted respectively during the tick blood meal, were collected from the bottom of both Petri dishes containing the two tick groups, to explore also the possibility for nymphs- 1 to become infected by direct contact with these excretions and/or secretions. 
Table 1. Proportions of ticks engorged during the co-feeding trial and real-time PCR results of ASFV and beta-actin detection. Results are shown as mean Ct [min Ct; $\max \mathrm{Ct}] . \mathrm{F}=$ female, $\mathrm{M}=$ male, $\mathrm{Ct}=$ Cycle threshold .

\begin{tabular}{|c|c|c|c|c|c|}
\hline Groups of Ticks & $\begin{array}{c}\text { Infected Adults (OmL) } \\
\text { Number of } \\
\text { Engorged/Total Adults }\end{array}$ & $\begin{array}{c}\text { Uninfected nymphs-1 } \\
\text { (OmCF) } \\
\text { Number of Engorged/Total } \\
\text { nymphs-1 }\end{array}$ & $\begin{array}{l}\text { Status of Pig } \\
\text { Used for Trial }\end{array}$ & $\begin{array}{l}\text { ASFV Detection in } \\
\text { Engorged Nymphs (Ct) }\end{array}$ & $\begin{array}{l}\text { Beta-actin Detection in } \\
\text { Engorged Nymphs }(\mathrm{Ct})\end{array}$ \\
\hline 1 & F: $6 / 8 ; \mathrm{M}: 5 / 7$ & $30 / 30$ & \multirow[t]{2}{*}{ Healthy } & No Ct & $\begin{array}{c}30,77 \mathrm{Ct} \\
{[28.74 \mathrm{Ct} ; 32.24 \mathrm{Ct}]}\end{array}$ \\
\hline 2 & F: $7 / 9 ; \mathrm{M}: 4 / 6$ & $28 / 30$ & & No Ct & $\begin{array}{c}30,09 \mathrm{Ct} \\
{[28.96 \mathrm{Ct} ; 31.55 \mathrm{Ct}]}\end{array}$ \\
\hline Control (OmCTR) & Not present & $11 / 11$ & $\begin{array}{c}\text { Infected } \\
\text { (Liv13/33 strain, } \\
10^{7.8} \mathrm{HAD}_{50} / \mathrm{mL} \text { ) }\end{array}$ & $\begin{array}{c}23,62 \mathrm{Ct} \\
{[23.06 \mathrm{Ct} ; 24.71 \mathrm{Ct}]}\end{array}$ & $\begin{array}{c}30,86 \mathrm{Ct} \\
{[30.29 \mathrm{Ct} ; 31.5 \mathrm{Ct}]}\end{array}$ \\
\hline
\end{tabular}


As a positive control to ensure the ability of $O$. moubata nymphs- 1 to become infected through the blood meal, a third group of uninfected O. moubata nymphs-1 (OmCTR), containing 11 specimen of OmCTR ticks were engorged on the same experimental pig when it became viremic (with a viremia of $10^{7.8} \mathrm{HAD}_{50} / \mathrm{mL}$ ) after the engorgement of infected ticks with the Liv13/33 strain.

A specific real-time polymerase chain reaction (PCR) for the detection of the ASFV VP72 gene [25] and beta-actin of ticks was used on individual DNA extracts from both tick samples, OmCF and OmCTR, two months after the co-feeding trial, as previously described [24]. Tick excretions and/or secretions collected from the Petri dishes were also tested for viral genome detection. The results were expressed as the cycle threshold $(\mathrm{Ct})$ for a qualitative analysis, to define the positive or negative status of the tick samples for ASFV.

A statistical analysis was carried out with RStudio (Version 1.1.463). A Kruskal test was used to compare the amplification of beta-actin, a tick housekeeping gene used as an internal control for DNA extraction quality, between the three tick groups.

\section{Results and Discussion}

As a result of the engorgement of adult OmL during the co-feeding test, the pig became infected by ASFV in two days. For the ticks, no statistical difference was found for the beta-actin results between OmCTR and groups 1 and 2 of OmCF ( $p$-value $=0.596)$, indicating that DNA extractions were homogeneous and comparable. All ticks of the OmCTR group were positive for ASFV (mean $\mathrm{Ct}=23.62$ [23.06-24.71]) (Table 1), confirming that nymphs-1 could be infected by the Liv13/33 ASFV strain through an infectious blood meal. Conversely, all OmCF ticks were negative for ASFV (No Ct) (Table 1), indicating the failure of ASFV co-feeding transmission between infected O. moubata adults and uninfected O. moubata nymphs- 1 . However, the ASFV genome was detected in the tick excretions and/or secretions collected from the Petri dishes of groups 1 and 2, where OmL and OmCF ticks were mixed, with real-time PCR results for ASFV detection of $28.20 \mathrm{Ct}$ and $29.14 \mathrm{Ct}$ for groups 1 and 2, respectively. This confirmed that adult ticks were infectious but could not infect nymphs-1 by direct contact with their infectious fluids.

The experimental design defined in the study was able to guarantee the reliability of the results obtained. Indeed, to maximize the success of transmission we used the African tick species O. moubata, which is known to acquire the Liv13/33 virus strain in salivary glands after infectious engorgement [21] and to transmit this virus to domestic pigs through biting [24]. To mimic as much as possible the natural conditions leading to tick infection through co-feeding [26], the transmission trial was carried out in small Petri dishes conducive to natural soft tick aggregation during the blood meal [27]. Naive nymphs-1 engorged synchronously with infected adults on uninfected pigs, as observed in natural soft tick populations.

Our results completed those obtained on pigs by Rennie (1998) [21], who suggested a failure of co-feeding transmission with the O. moubata-Liv13/33 tick-virus pair. However, her experimental design was quite different, since she used adult stages for both the infected "source" and the uninfected "recipient" ticks and separated them on the pig with a cardboard barrier inside the feeding unit to distinguished them after blood feeding. Interestingly, in a second trial, Rennie (1998) carried out co-feeding on mice, which is a non-sensitive animal for ASFV, and that time transmission occurred [21]. In this experiment, ticks were no longer separated but marked with a non-toxic light paint, and the only major difference from our study was the choice of the vertebrate host for blood feeding. However, Rennie attributed the successful infection to direct contact with infectious "source" ticks' coxal fluid secreted during the blood meal, which is apparently incongruent with our results. Therefore, ASFV co-feeding transmission between ticks may depend on the nature of the vertebrate host. Indeed, events that occur directly after tick bites (immunological responses and the spread of the pathogen in the vertebrate host) may impact co-feeding success and should be further investigated. In our experimental conditions, although the pig became sick 2 days after the engorgement of the infected adult ticks, which confirmed 
a substantial quantity of virus injected by the ticks, it is still possible to assume that this quantity was not sufficient to infect nymphs- 1 by co-feeding.

Taking our results and those of Rennie together, we can suggest that ASFV co-feeding cannot occur in domestic pigs using the O. moubata-Liv13/33 tick-virus pair, but additional experiments are needed to confirm these results. Co-feeding studies with others tick development stages are also needed to take into account the impact of the difference in hypostome (mouthparts) length. This difference may have an impact on the depth of the bite, and the nymphs- 1 could be unable to acquire the virus secreted by the infected adults during the blood meal. Warthogs, which are susceptible to ASFV, but which develop no or low viremia when they are infected, might mediate ASFV in the same manner as domestic pigs and may not be a major route for tick infection through co-feeding in the wild. Conversely, it is not possible to exclude the role of non-susceptible animals that may colonize ancient warthog burrows where soft ticks still settle, thereby contributing to tick infection through co-feeding and thus the persistence of the virus in tick populations. ASFV maintenance in soft ticks in the field is one cause of ASFV re-emergence [28]. However, the co-feeding transmission pathway has mainly been described in hard ticks, which remain attached to their host for days for blood feeding, while soft ticks can feed in less than an hour. The duration of the blood meal might also make co-feeding transmission less likely to happen in soft tick species.

Author Contributions: Investigation, R.P.D.O. and E.H.; methodology, F.P. resources, M.D.; conceptualization, M.-F.L.P. and L.V.; validation, M.-F.L.P. and LV; supervision, L.V.; writing-original draft, R.P.D.O.; writing-review and editing, R.P.D.O., E.H., M.D., F.P., M.-F.L.P., and L.V. All authors have read and agreed to the published version of the manuscript.

Funding: This research was funded by the National Institute of Food and Agriculture in the United States (grant $n^{\circ}$ 2019-67015-28981).

Acknowledgments: The authors are grateful to Jean-Marie Guionnet and Angélique Moro for animal care and to Peter Biggins for editing the English. Thanks to Vectopole Sud for funding the insectary where Ornithodoros moubata were raised and to the Direction Générale de l'Alimentation (Framework Convention SPA- 17 n 0079-E) and the National Institute of Food and Agriculture in the United-States (grant $n^{\circ}$ 2019-67015-28981) for the financial support to CIRAD to conduct scientific activities on ASF. We are thankful to CIRAD and ANSES for funding the PhD grant of Rémi Pereira De Oliveira.

Conflicts of Interest: The authors declare no conflict of interest. The funders had no role in the design of the study; in the collection, analyses, or interpretation of data; in the writing of the manuscript, or in the decision to publish the results.

\section{References}

1. Bakkes, D.K.; De Klerk, D.; Latif, A.A.; Mans, B.J. Integrative taxonomy of Afrotropical Ornithodoros (Ornithodoros) (Acari: Ixodida: Argasidae). Ticks Tick-Borne Dis. 2018, 9, 1006-1037. [CrossRef] [PubMed]

2. Boshoff, C.I. African swine fever virus maintenance and transmission dynamics in the sylvatic Ornithodoros vector. Ph.D. thesis, University of Pretoria, Pretoria, South Africa, 2017.

3. Haresnape, J.M.; Mamu, F.D. The distribution of ticks of the Ornithodoros moubata complex (Ixodoidea: Argasidae) in Malawi, and its relation to African swine fever epizootiology. Epidemiol. Infect. 1986, 96, 535-544. [CrossRef]

4. Plowright, W.; Parker, J;; Pierce, M.A. The epizootiology of African swine fever in Africa. Vet. Rec. 1969, 85, 668-674.

5. Jori, F.; Vial, L.; Penrith, M.L.; Pérez-Sánchez, R.; Etter, E.; Albina, E.; Michaud, V.; Roger, F. Review of the sylvatic cycle of African swine fever in sub-Saharan Africa and the Indian ocean. Virus Res. 2013, 173, 212-227. [CrossRef] [PubMed]

6. Penrith, M.-L.; Vosloo, W.; Jori, F.; Bastos, A.D.S. African swine fever virus eradication in Africa. Virus Res. 2013, 173, 228-246. [CrossRef] [PubMed]

7. Penrith, M.; Bastos, A.D.; Etter, E.M.C.; Beltrán-Alcrudo, D. Epidemiology of African swine fever in Africa today: Sylvatic cycle versus socio-economic imperatives. Transbound. Emerg. Dis. 2019, 66, 672-686. [CrossRef]

8. Thomson, G.R. The epidemiology of African swine fever: The role of free-living hosts in Africa. Onderstepoort J. Vet. Res. 1985, 52, 201-209. 
9. Plowright, W.; Perry, C.T.; Peirce, M.A.; Parker, J. Experimental infection of the argasid tick, Ornithodoros moubata porcinus, with African swine fever virus. Arch. Gesamte Virusforsch. 1970, 31, 33-50. [CrossRef]

10. Plowright, W. Vector transmission of African Swine Fever Virus. In Hog cholera/classical swine fever and African swine fever, Hannover (Germany, FR); Office for Official Publications of the European Communities: Brussels, Belgium, 1977.

11. Thomson, G.R.; Gainaru, M.D.; Van Dellen, A.F. Experimental infection of warthos (Phacochoerus aethiopicus) with African swine fever virus. Onderstepoort J. Vet. Res. 1980, 47, 19-22.

12. Rennie, L.; Wilkinson, P.J.; Mellor, P.S. Transovarial transmission of African swine fever virus in the argasid tick Ornithodoros moubata. Med. Vet. Entomol. 2001, 15, 140-146. [CrossRef]

13. Plowright, W.; Perry, C.T.; Greig, A. Sexual transmission of African swine fever virus in the tick, Ornithodoros moubata porcinus, Walton. Res. Vet. Sci. 1974, 17, 106-113. [CrossRef]

14. Randolph, S.E. Transmission of tick-borne pathogens between co-feeding ticks: Milan Labuda's enduring paradigm. Ticks Tick-Borne Dis. 2011, 2, 179-182. [CrossRef] [PubMed]

15. Randolph, S.E.; Gern, L.; Nuttall, P.A. Co-feeding ticks: Epidemiological significance for tick-borne pathogen transmission. Parasitol. Today 1996, 12, 472-479. [CrossRef]

16. Labuda, M.; Kozuch, O.; Zuffová, E.; Elecková, E.; Hails, R.S.; Nuttall, P.A. Tick-Borne Encephalitis Virus Transmission between Ticks Cofeeding on Specific Immune Natural Rodent Hosts. Virology 1997, 235, 138-143. [CrossRef] [PubMed]

17. Jones, L.; Davies, C.; Steele, G.; Nuttall, P. A novel mode of arbovirus transmission involving a nonviremic host. Science 1987, 237, 775-777. [CrossRef]

18. Labuda, M.; Jones, L.D.; Williams, T.; Danielova, V.; Nuttall, P.A. Efficient Transmission of Tick-Borne Encephalitis Virus Between Cofeeding Ticks. J. Med. Entomol. 1993, 30, 295-299. [CrossRef]

19. Gordon, S.W.; Linthicum, K.J.; Moulton, J.R. Transmission of Crimean-Congo hemorrhagic fever virus in two species of Hyalomma ticks from infected adults to cofeeding immature forms. Am. J. Trop. Med. Hyg. 1993, 48, 576-580. [CrossRef]

20. Belli, A.; Sarr, A.; Rais, O.; Rego, R.O.M.; Voordouw, M.J. Ticks infected via co-feeding transmission can transmit Lyme borreliosis to vertebrate hosts. Sci. Rep. 2017, 7, 1-13. [CrossRef]

21. Rennie, L.F. The persistance of African Swine Fever Virus in the Argasid Tick Ornithodoros Moubata, University of Hertfordshire. 1998. Available online: https://ethos.bl.uk/OrderDetails.do?uin=uk.bl.ethos. 245350 (accessed on 1 April 1998).

22. Dixon, L.K.; Wilkinson, P.J. Genetic Diversity of African Swine Fever Virus Isolates from Soft Ticks (Ornithodoros moubata) Inhabiting Warthog Burrows in Zambia. J. Gen. Virol. 1988, 69, 2981-2993. [CrossRef]

23. Bernard, J.; Hutet, E.; Paboeuf, F.; Randriamparany, T.; Holzmuller, P.; Lancelot, R.; Rodrigues, V.; Vial, L.; Le Potier, M.-F. Effect of O. porcinus Tick Salivary Gland Extract on the African Swine Fever Virus Infection in Domestic Pig. PLoS ONE 2016, 11, e0147869. [CrossRef]

24. Pereira de Oliveira, R.; Hutet, E.; Paboeuf, F.; Duhayon, M.; Boinas, F.; Perez de Leon, A.; Filatov, S.; Vial, L.; Le Potier, M.-F. Comparative vector competence of the Afrotropical soft tick Ornithodoros moubata and Palearctic species, O. erraticus and O. verrucosus, for African swine fever virus strains circulating in Eurasia. PLoS ONE 2019, 14, e0225657. [CrossRef]

25. Tignon, M.; Gallardo, C.; Iscaro, C.; Hutet, E.; Van der Stede, Y.; Kolbasov, D.; De Mia, G.M.; Le Potier, M.-F.; Bishop, R.P.; Arias, M.; et al. Development and inter-laboratory validation study of an improved new real-time PCR assay with internal control for detection and laboratory diagnosis of African swine fever virus. J. Virol. Methods 2011, 178, 161-170. [CrossRef] [PubMed]

26. Voordouw, M.J. Co-feeding transmission in Lyme disease pathogens. Parasitology 2015, 142, 290-302. [CrossRef]

27. Hamilton, J.G.C. The role of pheromones in tick biology. Parasitol. Today 1992, 8, 130-133. [CrossRef]

28. Boinas, F.S.; Wilson, A.J.; Hutchings, G.H.; Martins, C.; Dixon, L.J. The Persistence of African Swine Fever Virus in Field-Infected Ornithodoros erraticus during the ASF Endemic Period in Portugal. PLoS ONE 2011, 6, e20383. [CrossRef] [PubMed]

(C) 2020 by the authors. Licensee MDPI, Basel, Switzerland. This article is an open access article distributed under the terms and conditions of the Creative Commons Attribution (CC BY) license (http://creativecommons.org/licenses/by/4.0/). 